\title{
Jay P. Corrin, Catholic Progressives in England after
} Vatican II

Notre-Dame (Indiana), University of Notre Dame Press, 2013, X-536 p. Notes bibliographiques, index

\section{Paul Airiau}

\section{(2) OpenEdition}

\section{Journals}

Édition électronique

URL : http://journals.openedition.org/assr/27333

DOI : $10.4000 /$ assr. 27333

ISSN : $1777-5825$

Éditeur

Éditions de l'EHESS

Édition imprimée

Date de publication : 1 octobre 2015

Pagination : 282

ISBN : 978-2-7132-2515-4

ISSN : 0335-5985

Référence électronique

Paul Airiau, « Jay P. Corrin, Catholic Progressives in England after Vatican II 》, Archives de sciences sociales des religions [En ligne], 172 | octobre-décembre, mis en ligne le 19 juillet 2016, consulté le 23 septembre 2020. URL : http://journals.openedition.org/assr/27333 ; DOI : https://doi.org/10.4000/ assr.27333

Ce document a été généré automatiquement le 23 septembre 2020

(c) Archives de sciences sociales des religions 


\section{Jay P. Corrin, Catholic Progressives in England after Vatican II}

Notre-Dame (Indiana), University of Notre Dame Press, 2013, X-536 p. Notes bibliographiques, index

\section{Paul Airiau}

\section{RÉFÉRENCE}

Jay P. Corrin, Catholic Progressives in England after Vatican II, Notre-Dame (Indiana), University of Notre Dame Press, 2013, X-536 p., Notes bibliographiques, index. 
$1 \quad$ Est-il possible d'écrire trois livres en un? Sans doute, puisque c'est ce que tente de faire Jay P. Corrin, professeur de sciences sociales à Boston University, auteur de travaux sur le distributisme anglais (Gilbert Keith Chesterton, Hilaire Belloc) et la "conversion" démocratique des intellectuels catholiques aux $\mathrm{XIX}^{\mathrm{e}}$ et $\mathrm{XX}^{\mathrm{e}}$ siècles. Pour tenir ensemble l'histoire sociopolitique des catholiques anglais des années 1900 aux années 1950 centrée sur le distributisme; une interprétation générale de Vatican II ; et le " gauchisme » catholique anglais des années 1960 (on excusera l'utilisation de ce terme très français de "gauchisme ", mais il est fort approprié à ce qui est raconté), il faut malgré tout une problématique commune. Il paraît cependant difficile d'articuler ces

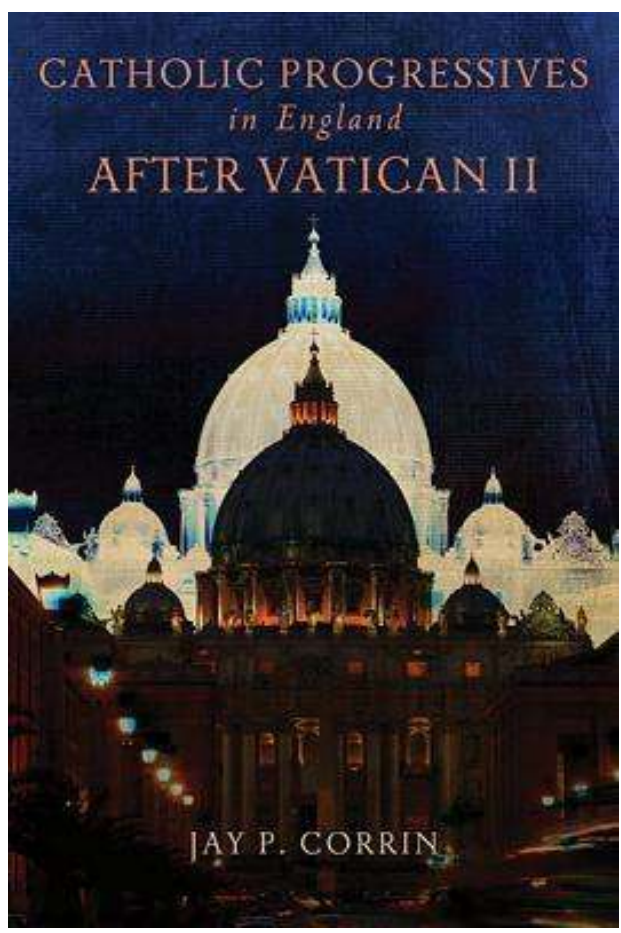
trois parties du livre, même si l'on conçoit bien que l'auteur ait voulu poser un contexte général, montrer le rôle transformateur de Vatican II, et observer alors la bouffée gauchiste catholique anglaise des années 1960-1970. C'est pourtant prendre les choses de fort loin que de consacrer une première partie de cinquante pages au cadre catholique anglais du premier $\mathrm{xx}^{\mathrm{e}}$ siècle, puis de traiter la poussée réformatrice mondiale aboutissant à Vatican II en une deuxième partie de presque 110 pages, avant d'entrer en profondeur dans une troisième et dernière partie de plus de 200 pages étudiant le périodique Slant, ses idées et son réseau.

2 Outre cette interrogation sur la construction générale, voire sur la construction plus précise de la troisième partie (loin de suivre un cours chronologique, J. P. Corrin y fait des allers et retours rendant parfois délicate l'exposition ordonnée de l'objet d'étude, car il privilégie l'étude des idées développées par les "catholiques progressistes », ce qui tend à donner une succession d'études thématiques où le lien organique est réduit), il est difficile de ne pas s'interroger également sur la rigueur mise en œuvre. Passons sur les noms mal orthographiés : Johann Joseph Iznaz von Döllinger au lieu de Ignaz, p. 67 ; Giovanni Montini p.68, Giovanni Battista Montini p. 137, Giovanni Baptista Montini ; Pio Nino au lieu de Pio nono, p. 101 (pourquoi par Pio IX tout simplement ?) ; le P. Garrigou-Legrange au lieu de Garrigou-Lagrange, p. 81-82; Benoît XVII et non Benoît XVI, p. 299. Mais il est des erreurs factuelles étonnantes : l'apostasie de Döllinger fondant la secte des vieux catholiques (p. 67 - les qualificatifs d'apostasie et de secte, tels qu'ils sont ici utilisés, relèvent plus du vocabulaire de l'orthodoxie doctrinale et ecclésiologique que de la sociologie ou de l'histoire) ; la domination du thomisme dans l'enseignement catholique depuis le Moyen Âge (p.67), alors que Léon XIII établit Thomas d'Aquin comme penseur officiel du catholicisme contre d'autres pensées et malgré de nombreuses résistances; la fin de l'interdit sur les écrits du P. Teilhard de Chardin après sa mort (p. 93), alors qu'un monitum du Saint-Office met encore en garde contre le jésuite mort en 1962 ; la fondation de Concilium en 1962-1963 (p. 139), au lieu 
de 1965 ; Études devenue une revue dominicaine (p. 80), au lieu de jésuite ; une Nouvelle théologie (sans guillemets, mais avec majuscule) culminant dans le mouvement des prêtres ouvriers (p.83), alors que ceux-ci ont peu de rapports avec les jésuites de Fourvière principalement visés lors de la querelle de la "théologie nouvelle"; Mgr Ottaviani déjà cardinal en 1951 (p. 84), au lieu de 1953 ; les dirigeants de l'Église de France plus soucieux, après le départ de Mgr Roncalli, de défendre les industriels et le gouvernement qui garantissent la loi et de l'ordre (p. 84), ce qui empêche de comprendre pourquoi ils intervinrent à Rome en 1954 pour éviter toute remise en cause des prêtres-ouvriers; la signature de concordats par Pie XI avec l'Italie, le Mexique, l'Espagne, l'Allemagne, qui échangeaient la neutralité des catholiques contre un statut de religion d'État (p. 109), alors que le catholicisme ne fut pas religion d'État en Allemagne en 1933, qu'il fallut attendre 1959 pour qu'un concordat fût signé avec l'Espagne, et, quant au Mexique, où la politique anticléricale du président Calles suscita un soulèvement armé d'une partie des populations catholiques de 1926 à 1929 (les Cristeros), l'avenir le dira; l'ambiance de terreur curiale nécessitant l'utilisation d'un pseudonyme par le P. Francis X. Murphy durant Vatican II pour jouer son rôle d'informateur religieux (p.134), alors que la Curie fut dès l'ouverture du concile incapable de contrôler la circulation des informations; l'adoption de Lumen Gentium le 11 novembre 1964 (p. 140), au lieu du 21 ; l'introduction des langues vernaculaires à la place du latin dans Sacrosanctum consilium (p. 143), alors que l'usage du latin y demeure la norme et le vernaculaire une exception encadrée (\$36); le «cercle thomiste » de Maritain (p. 238), au lieu des cercles thomistes; Gaudium et spes qualifié d'encyclique (p. 246, 288), au lieu de constitution ; la prise de Rome par les forces italiennes sur les troupes françaises en 1870 (note 27 p. 401), alors que celles-ci s'en étaient retirées début août et que la conquête se fit contre les troupes pontificales le 20 septembre 1870 ; Catholicisme : les aspects sociaux du dogme daté de 1951 (note 41 p. 446), alors que la première édition française date de 1938, la quatrième édition revue et augmentée de 1947, la cinquième de 1952, les premières éditions anglaise et états-unienne, sous le titre Catholicism. A Study of Dogma in Relation to the Corporate Destiny of Mankind, étant de 1950 (London, Burns, Oates \& Washbourne ; New York, Longmans, Green ou Sheed and Ward).

3 Certes, nul n'est à l'abri d'erreurs, de fautes de copie, de vérifications fondées sur des sources défectueuses, d'un travail éditorial déficient (le Benoît XVII déjà évoqué, ou le Paul XI auteur de Quadragesimo anno, p. 71), ou du travail trop rapide d'un ancien étudiant se penchant sur Vatican II pour son ancien professeur - quand bien même celui-ci revendique que " any omission and errors that remain in this work are enterily [his] own » (p. X). Mais lorsqu'en plus de cette accumulation d'erreurs factuelles, il est des absences bibliographiques décevantes, cela devient beaucoup. Ainsi spécialement pour la deuxième partie, sur Pie XII, Jean XXIII et Vatican II. L'Histoire de Vatican II dirigée par Giuseppe Alberigo est absente (alors qu'est présente en bibliographie la version anglaise du collectif qu'il dirigea en 1985 avec Jean-Pierre Jossua [et non Jussua] et Joseph A. Komonchak, La réception de Vatican II), bien que son édition états-unienne ait été publiée de 1995 à 2006 (Maryknoll, NY, Orbius, et Leuven, Peeters). Pie XII n'est abordé qu'à travers John Cornwell, Philippe Chenaux passant à la trappe, et Jean XXIII que par Peter Hebblethwaite, alors qu'Étienne Fouilloux a édité le journal du nonce Roncalli. Quant aux travaux consacrés à l'histoire intellectuelle du catholicisme européen, pourtant abondamment présentée, ils n'existent pas. Étienne Fouilloux semble n'avoir jamais rien écrit sur les années 1914-1960, ni sur la nouvelle théologie. 
Plus généralement, c'est toute l'historiographie européenne non traduite en anglais qui est laissée de côté. Certes, nul n'est obligé de connaître toutes les langues. Mais il est toujours possible de se donner les moyens d'avoir accès à des références indispensables, même lorsqu'a priori le sujet travaillé (le catholicisme anglais) paraît peu concerné par les réalités continentales (c'est-à-dire européennes) - a priori, bien sûr, car si la Grande-Bretagne est irréductiblement insulaire, les mers se traversent, et les livres se traduisent: la maison Sheed and Ward s'est chargée dans les années 1950-1960 de proposer aux lecteurs anglais la théologie continentale, et si J. P. Corrin le rappelle, il n'en tire pas de conséquences. N'évoquons même pas la présence du pamphlet antijésuite de l'ancien jésuite au complexe parcours Malachi Martin, The Jesuits: The Society of Jesus and the Betrayal of the Roman Catholic Church, datant de 1987 (note 55 p. 441, utilisé comme source d'information à propos du jésuite états-unien James Carney, mort en 1983 dans les rangs de la guérilla marxisante au Honduras - et vraisemblablement sans avoir été lui-même combattant, dans des circonstances bien plus incertaines que ce que rapporte Corrin en se fondant sur Martin).

4 Tout cela est d'autant plus gênant que cela se conjugue à une surinterprétation et une tendance à grossir le trait. Certes, on peut s'expliquer par une pratique anglo-saxonne voulant rendre « attrayante » l'histoire ou les sciences sociales. Un lecteur continental reconnaît très vite ce ton particulier du lettré anglais ou américain qui, loin de la teutonne érudition ou de la pesante précision française, pratique le décalage, l' understatement, la lecture au deuxième degré, l'héroïsation de la banalité, le marivaudage intellectuel, la mise en scène et la théâtralisation des distinctions, oppositions et enjeux, etc. Mais il faut avouer que les nécessaires nuances à la compréhension fine de la réalité disparaissent. Ainsi, la comparaison entre Pie XII et Jean XXIII se fait à traits forcés, qu'il s'agisse de la conception quasi médiévale que Pie XII avait de son mode de gouvernement (p. 68-69 - que dire alors de ce que fit Pie XI ?) ou de la volonté de Jean XXIII d'aborder les questions temporelles brûlantes comme la Guerre froide, la prolifération nucléaire et l'idéologie communiste (p.119), comme si Pie XII ne les avait jamais traitées, comme si Divini redemptoris de Pie XI (1937 - Eugenio Pacelli était alors Secrétaire d'État...) n'était pas un jugement sur l'idéologie communiste. La «nouvelle théologie » devient une «coterie» (p. 74) au service d'une conception originelle du christianisme, c'est-à-dire sociale: «renforcer la mission humaniste de l'Église pour améliorer l'organisation sociale et politique de la société » (p. 74). Même, elle devient l'ensemble des mouvements alternatifs au thomisme romain dans les années 1950, allant jusqu'à comprendre les prêtres ouvriers, on l'a dit, mais aussi Hans Küng et Pierre Teilhard de Chardin (p. 70-87), les deux héros de la seconde partie, le premier servant de guide sûr au travers de la dynamique conciliaire - autant raconter la Révolution française en ne se fondant que sur les assertions d'un de ses acteurs, disons l'abbé Barruel ou Joseph Fouché... - et le second d'exemple paradigmatique à une pensée catholique moderne et progressiste, harmonisant l'amour de Dieu et l'amour des hommes, et remettant en cause la revendication d'unicité de l'Église catholique et son fonctionnement autocratique (p. 74-83). Quant à Vatican II, quel récit trouve-t-on si ce n'est celui qui en fait la lutte entre la Curie réactionnaire et les évêques réformateurs, même si, au passage, on relève l'affirmation que le concile est, à suivre l'historien anglais Adrian Hastings, "déchiré entre "conservateurs réactionnaires" et "conservateurs libéraux" avec très peu de vrais radicaux » (p. 135).

5 J. P. Corrin propose finalement une interprétation de l'histoire où les appréciations sont incohérentes. Deux exemples simples l'illustrent. Alors que Pie XII est décrit 
comme un autocrate ignorant les cardinaux et traitant ses collaborateurs comme des laquais (p. 66-69), devenu vieux, il cède à la pression des conservateurs de la Curie pour exiler Montini à Milan (p. 140). De même, Pie XI condamne le communisme avec Divini Redemptoris et Pie XII, par peur du communisme, menace d'excommunication tout catholique rejoignant le PC ou votant pour ses candidats (p. 89), mais il faut attendre Jean XXIII pour se saisir de cette question brûlante (p.119). Comment tenir ces faits ensemble? Comment harmoniser la présentation presque louangeuse des distributistes avec leur antilibéralisme économique forcené et leur rejet absolu du marxisme, qui tranche avec la conformité des catholiques anglais englués dans la société bourgeoise et libérale dans les années 1920 (p. 21-41), et la valorisation du marxisme catholique de Slant, en rupture radicale avec des catholiques anglais présentés comme toujours aussi conservateurs politiquement et socialement? Comment émettre l'idée (le regret ?) que des catholiques marxisés des années 1960 auraient pu rechercher dans le distributisme des années 1920-1930 une ressource intellectuelle pour élargir leur audience et légitimer leurs prises de position, alors que leurs pensées, quoique conjointement anticapitalistes et quoi qu'elles présentent des parallélismes dans la critique du libéralisme économique et politique et la volonté de créer des communautés alternatives, sont objectivement incompatibles (p.41)? Au final, serait-ce donc l'antilibéralisme économique et la volonté de transformation sociale contre les puissances de l'argent et la bourgeoisie, qui seraient l'important et donc le critère de jugement implicite utilisé par J.P. Corrin? Ainsi s'expliquerait que puissent être présentés aussi favorablement et l'antilibéralisme intransigeant dans les années 1920 et l'anlibéralisme marxisé dans les années 1960, et que soit tenue pour pas grand-chose la participation catholique (irlandaise) aux trade unions et le cardinal Manning pour une exception.

6 Mais alors le souci principal de J. P. Corrin serait de défendre une conception de ce que doit être le positionnement catholique dans le monde contemporain : un engagement sociocivique, sans exclusives politiques, pour la libération de l'homme. D'où la lecture négative de la situation catholique britannique d'avant Vatican II, présentée comme une sous-culture conformiste, politiquement et socialement, et de l'Église catholique d'avant Jean XXIII, ramenée à l'image d'une institution conservatrice, en conflit avec le monde, refusant d'écouter les requêtes modernes. Pourtant, tout cela, c'est au risque de ne pas percevoir et comprendre ce que l'historiographie continentale (française et italienne, spécialement) a bien établi : l'Église antérieure à Jean XXIII n'entretenait pas seulement une relation conflictuelle avec la société, mais développait aussi, depuis le milieu du $\mathrm{XIX}^{\mathrm{e}}$ siècle au moins, une compétition/rivalité/contestation suscitant une dynamique d'engagement social (y compris chez les opposants à la démocratie, et pas seulement chez Frédéric Ozanam, présenté comme une exception, p. 178-179), bientôt voulue par les papes, au service de la réforme sociale (et non de la révolution) par la catholicisation, au risque de constituer une contre ou alter-société. À cet égard, l'absence du mouvement catholique amorcé par la "génération Léon XIII", et l'ignorance de l'Action catholique de Pie XI, sont typiques - et consternantes. Et l'insularité de la Grande-Bretagne ne pourrait être un argument suffisant pour considérer que le catholicisme y a ignoré des dynamiques qui sont mondiales - J.P. Corrin lui-même souligne la romanisation des prélats anglais (p. 12).

7 Lire Émile Poulat, Yvon Tranvouez et Jean-Marie Mayeur aurait été profitable à J. P. Corrin. Les choses lui eussent sans doute alors paru moins étranges. Il aurait mieux compris comment un même catholicisme peut donner naissance, dans les années 
1920-1930, au distributisme, et dans les années 1960, à des progressistes marxisés ; comment un catholicisme numériquement marginal, marqué par une bipolarité nationale (irlandaise et anglaise) et modelé selon l'intransigeance pontificale (dans les rapports avec le monde et en ecclésiologie), peut tout à fait logiquement développer une contre-société cléricale (comme ce qui se passa aux États-Unis et au Québec des années 1880 aux années 1960); comment l'essor numérique et proportionnel des années 1900-1970 peut amener des transformations internes importantes, surtout lorsqu'elles sont conjuguées à des mutations fortes de la part du système intransigeant. Cela aurait enfin permis de prendre plus de distance avec les sources primaires: les souvenirs des membres de Slant servent bien souvent de grille d'analyse (notamment sur ce qu'était le catholicisme anglais des années 1950, chapitre 1).

Bref, il aurait pu au final mieux éclairer la question qui le taraude, celle des relations entre le distributisme et Slant. Car il hésite longuement (spécialement p. 363-368) : le distributisme est une « racine profonde d'un radicalisme catholique anglais » (p. 41); il y a une filiation entre les deux courants, quoiqu'elle n'ait été explicitement revendiquée au sein de Slant que par Adrian Cunningham à propos d'Eric Gill, l'artisteartisan distributiste des années 1900-1930 (p. 366 - la biographie de Fiona MacCarthy, Eric Gill, London, Faber and Faber, 1989, est absente de la bibliographie); Slant est le « développement ultérieur [au distributisme] d'un côté radical de la pensée catholique sociale » (p. 363); il y a de " proches relations » et une « résonance considérable » entre les deux mouvements (p. 364). Pour éclairer complètement la question, il aurait fallu étudier le distributisme comme modalité du catholicisme intransigeant, et en faire l'histoire jusque dans les années 1940-1965 (il est expédié en quelques phrases, p. 40-41, $363,367)$. La rupture humaine aurait sans doute apparu, mais la dimension matricielle $\mathrm{du}$ catholicisme intransigeant aurait permis de comprendre comment les transformations socio-économiques et les évolutions historiques en font surgir des actualisations-adaptations spécifiques qui perdent la conscience de leur commune origine.

9 Malgré tout, il est possible de tirer de l'ouvrage, en le lisant la plume à la main et en recomposant l'histoire qui n'est pas racontée, mais fragmentée entre les chapitres et les notes de la troisième partie, un certain nombre d'éléments sur le catholicisme socialiste anglais des années 1960-1970, à la lumière du groupe Slant. Celui-ci s'enracine dans le contexte de la fin des années 1950, avec l'émergence de la New Left, associée à la remise en cause du soviétisme d'après Budapest et le $\mathrm{XX}^{\mathrm{e}}$ congrès du PCUS et en dissonance avec un Labour trop acculturé à un réformisme progressif éloigné de l'anticapitalisme, et avec le fort mouvement pacifiste antinucléaire de 1957-1963. Son terreau social est l'émergence d'une nouvelle jeune élite intellectuelle catholique profitant de l'ouverture scolaire et universitaire de l'après-Seconde Guerre mondiale, notamment à Cambridge ou Oxford : Terry Eagleton, Neil Middleton, Adrian Cunningham, Martin Shaw, Bernard Sharrat, Christopher Colnan, Leo Pyle. Son humus intellectuel et ecclésial est l'ordre dominicain anglais, avec en particulier Laurence Bright, Herbet McCabe et Fergus Kerr, ainsi que le December Group, groupe de réflexion catholique sur les questions sociales apparu au début des années 1960, et la maison d'édition Sheed and Ward. En 1964, ces jeunes universitaires fondent le trimestriel Slant, devenu un bimestriel lorsque l'édition est assurée par Sheed and Ward à partir de 1966, et ce jusqu'en 1970. À son apogée, la publication aura 200 à 300 abonnés et écoulera jusqu'à 2000 numéros. L'objectif est de croiser le catholicisme et le marxisme pour transformer l'Église catholique britannique, conformiste et bourgeoise, en une communauté démocratique du peuple de Dieu 
faisant surgir une nouvelle culture et engagée pour libérer l'homme de l'aliénation capitaliste. Plus que le marxisme "orthodoxe", les jeunes intellectuels de Slant s'abreuvent à l'hétérodoxie marxiste des années 1950-1970 (le jeune Marx, Lukacs, Gramsci...) et aux sciences humaines en plein essor. Ils soutiennent tous les mouvements catholiques ou sociaux susceptibles de remettre en cause l'ordre bourgeois et le fonctionnement de l'Église catholique - mais en ignorant la « libération des corps ": à cet égard, on peut se demander si, en négligeant tout le freudomarxisme, ils ne signent pas leur profond enracinement catholique. Ainsi, lors de l'affaire Charles Davis, théologien qui quitte le sacerdoce et le catholicisme à la fin de 1966, ils remettent en cause l'exercice hiérarchique du pouvoir puisque le P. McCabe a été sanctionné pour sa réponse à Davis (alors que ce dernier jugeait irréformable les structures catholiques, le dominicain utilisait la dialectique pour mettre les évêques à la marge du progrès humain et religieux, et les groupes réformateurs au centre). Leur approche très conceptuelle, illustrée notamment par un colloque organisé à Birmingham en 1965 (publié en 1966 sous le titre The Committed Church), une prise de position générale en 1966 (Slant Manifesto : Catholics and the Left), et un colloque en 1967 (From Culture to Revolution: The Slant Symposium, 1967, 1968), limite cependant leur audience au sein du catholicisme. Ils tentent de pallier leur faible efficacité en lançant un bulletin (Slant Bulletin puis Slant X) et des groupes locaux (environ 25 en 1968), et en participant à certaines des mobilisations des années 1960 : lutte contre la guerre du Vietnam, débats avec le PC anglais, soutien à l'agitation estudiantine de la London School of Economy, participation à la Blackleath Commune (une forme de communauté de base de 1969 à 1971), collaboration avec les catholiques socialistes d'Irlande. Le tout début des années 1970 voit Slant se déliter, en raison de la coupure entre le positionnement théorique des rédacteurs de la revue et les militants des groupes locaux, et du recadrage de la réception de Vatican II par Paul VI, qui signe pour eux l'échec conciliaire. Les rédacteurs, s'intégrant alors plutôt bien au monde universitaire anglais (Terry Eagleton, en particulier), s'éloignent à des degrés divers du catholicisme.

Nul doute que les historiens du catholicisme français retrouveront ici nombre d'éléments qui leur sont familiers quant à la question des rapports entre catholicisme et marxisme, à la fois avec une chronologie décalée d'une bonne dizaine d'années (le progressisme catholique français est consécutif à la Seconde Guerre mondiale, et il est lié lui aussi en partie à la question de la paix et de l'armement nucléaire) et des consonances temporelles (les effets de Vatican II, la nouvelle gauche française, le moment gauchiste des années 1960-1970, la rapidité des évolutions à partir de 1965). Nul doute aussi que le destin universitaire associé à l'éloignement de la pratique religieuse leur rappellera certains traits des élites catholiques formées dans les années 1960-1970. C'est donc au moins le mérite de l'ouvrage de pousser à souhaiter une approche comparatiste des " gauches catholiques » ou des « catholiques de gauche » en Europe, voire dans le monde, afin de percevoir les spécificités nationales, les synchronies mondiales ou continentales, et les circulations des hommes et des idées. À cet égard, on ne peut que se féliciter de l'organisation par le Centre d'Histoire de l'Institut d'études politiques et la fondation Jean Jaurès (Paris, 21-22 mai 2015) du colloque « L'esprit de Vatican II. Catholiques de gauche en Europe occidentale dans les années $60-70$ "*. À l'instar de toute une série de travaux menés depuis une dizaine d'années, ils montrent que le temps de l'historicisation universitaire de Vatican II et de sa réception est (enfin) arrivé - quand bien même cela traduit aussi bien sûr une interrogation de la gauche et d'une partie des catholiques de gauche sur les fruits de ce 
qu'ils ont âprement défendu: mais ceci est une autre histoire (sur laquelle divers chercheurs, dont Guillaume Cuchet, ont déjà commencé à s'interroger), et aussi la même histoire, tant, désormais, pour les historiens, les événements, leur réception et leur interprétation postérieure forment un tout indissociable.

$11{ }^{*}$ Le Bulletin bibliographique rendra évidemment compte de ces rencontres lors de la publication du volume attendu (NDLR). 